\title{
Increased Close Appositions between Corticospinal Tract Axons and Spinal Sympathetic Neurons after Spinal Cord Injury in Rats
}

\author{
BAOHAN PAN, ESTHER J. KIM, and LAWRENCE P. SCHRAMM
}

\begin{abstract}
Treatments for spinal cord injury may promote new spinal cord synapses. However, the potential for new synapses between descending somatomotor and spinal sympathetic neurons has not been investigated. We studied rats with intact spinal cords and rats after a chronic, bilateral, dorsal spinal hemisection. We identified sympathetically related spinal neurons by transynaptic, retrograde transport of renally injected pseudorabies virus. We counted retrogradely labeled sympathetic preganglionic neurons (SPN) and putative sympathetic interneurons (IN) that, under light microscopy, appeared closely apposed by anterogradely labeled axons of the corticospinal tract (CST) and by axons descending from the well-established sympathetic regulatory region in the rostral ventrolateral medulla (RVLM). Spinal sympathetic neurons that were closely apposed by CST axons were significantly more numerous in lesioned rats than in unlesioned rats. CST axons closely apposed 5.4\% of SPN and $10.3 \%$ of IN in rats with intact spinal cords, and $38.0 \%$ of SPN and $37.3 \%$ of IN in rats with chronically lesioned spinal cords. Further, CST appositions in SCI rats consisted of many more varicosities than those in uninjured rats. SPN and IN closely apposed by axons from the RVLM were not more numerous in lesioned rats. However, RVLM axons apposed many more SPN than IN in both control and lesioned rats. Therefore, RVLM sympathoexcitation may be mediated largely by direct synapses on SPN. Although we have not determined the functional significance of close appositions between the CST and spinal sympathetic neurons, we suggest that future studies of spinal cord repair and regeneration include an evaluation of potential, new, somatic-autonomic interactions.
\end{abstract}

Key words: axon collaterals; rostral ventrolateral medulla; spinal cord regeneration; sympathetic preganglionic neurons; sympathetically related interneurons

\section{INTRODUCTION}

$\mathbf{R}$ ECENTLY DEVELOPED STRATEGIES promise functional recovery after spinal cord injury (SCI). To date, most studies of SCI have concentrated on promoting regener- ation or sprouting of somatomotor pathways or on the reduction of damage subsequent to injury (Ramer et al., 2005). Less attention has been paid to the possibility that regenerating or sprouting axons might form inappropriate synapses leading to dysfunction. Inappropriate

Department of Biomedical Engineering, The Johns Hopkins School of Medicine, Baltimore, Maryland. 
synapses between somatomotor pathways and spinal autonomic neurons are likely to be particularly harmful.

Several observations lend credence to this concern. First, after transection, the corticospinal tract of the rat collateralizes abundantly into new regions of the dorsal horn and intermediate zone (Fouad et al., 2001). Second, sprouting corticospinal tract axons (CST) make new connections with somatic interneurons above incomplete spinal lesions. The axons of these interneurons, in turn, bypass the lesions and make new synapses caudally (Bareyre et al., 2004). That spinal synaptic plasticity may be associated with serious cardiovascular dysfunction is suggested by the observation by Llewellyn-Smith and Weaver (2002) of substantial plasticity of synapses on SPN correlated to the autonomic dysreflexia exhibited by rats after spinal cord lesions.

In addition to clinically related concerns, additional motivation exists for studying projections from the brain to spinal sympathetic neurons. First, few studies have investigated direct cortical projections to spinal sympathetic neurons. Second, although the projections to sympathetic preganglionic neurons (SPN) of the rostral ventrolateral medulla (RVLM) and the paraventricular nucleus of the hypothalamus have been well described (Ranson et al., 1998), brainstem projections to interneurons (IN) that are spinal synaptic antecedents of SPN have not.

The data obtained to address these issues were the degree of sprouting of CST axons and axons from the RVLM rostral to a spinal cord injury, and the number of SPN and IN that exhibited putative input from the sensorimotor cortex and RVLM. To this end, we anterogradely labeled the corticospinal tract (CST) or axons descending from the RVLM. We used the retrograde, transynaptic transport of renally injected pseudorabies virus (PRV) to label SPN that projected to renal, postganglionic, sympathetic neurons. This method also identified spinal IN that, in turn, projected to those infected "renal" SPN. To ensure the study of a coherent population, we quantified only SPN and IN infected after renal injections of PRV. In spinally intact rats and in rats surviving 1 month after an L2 dorsal spinal hemisection, we counted the SPN and IN that were closely apposed by CST axons and by axons projecting from the RVLM (as determined by light microscopy). Our criteria for identifying close appositions are described below. We recognize that close appositions only permit us to infer potential synaptic input to spinal sympathetic neurons. Definitive demonstration of synapses would require electron microscopic analysis. The technical difficulties involved in electron microscopic identification of synapses in multiply labeled tissue are examined below.

In spinally intact rats, the CST closely apposed few
SPN or IN. However, the CST collateralized abundantly rostral to spinal lesions, and many more SPN and IN were closely apposed by those collaterals. Many infected SPN (but significantly fewer infected IN) were closely apposed by axons projecting from the RVLM in rats with intact spinal cords. Axons descending from the RVLM did not sprout significantly after spinal lesions, and the number of infected SPN and IN closely apposed by those axons did not change after spinal lesions. These results have been presented in preliminary form (Pan et al., 2004).

\section{MATERIALS AND METHODS}

We conducted these experiments in adult, male, Sprague-Dawley rats (approximately 2 months old, 150-200 g; Charles River, Raleigh, NC). All surgical procedures and post-operative care were provided in accordance with the Guide for the Care and Use of Laboratory Animals (National Research Council, 1996) and approved by the Johns Hopkins University Committee on Animal Care and Use. We lesioned the spinal cords of 40 rats. Forty-five rats were used as unoperated controls. Twelve of the lesioned rats died or were sacrificed after surgery. Fifty-five rats were eliminated from our final analysis due to inadequacies of tracing methods.

\section{Spinal Cord Injury (SCI)}

We anesthetized rats with halothane (5\% induction, 1.5-2.5\% maintenance) and made a dorsal laminectomy at the level of the $\mathrm{T}_{13}$ and $\mathrm{L}_{1}$ vertebrae (corresponding to the $\mathrm{L}_{2}$ and $\mathrm{L}_{3}$ spinal segments). We incised the dura and hemisected the dorsal spinal cord at the $\mathrm{L}_{2-3}$ level with a micro-surgical blade (Fig. 1C). We inserted the blade $2.0 \mathrm{~mm}$ from the dorsal surface to extend the lesion ventral to the central canal. Muscle and skin incisions were closed separately. Rats were treated with an antibiotic (20,000 units, i.m.; Pfizerpen, Pfizer, NY), and an analgesic (1.1 mg/kg, s.c.; Banamine, Shering-Plough, Union, NJ) before cessation of anesthesia. We manually expressed rats' bladders for 3-5 days after surgery, by which time spontaneous micturition recovered.

\section{BDA Injections}

Two weeks after rats were spinally lesioned, we divided the lesioned and control rats into two groups. We injected the sensorimotor cortices of the first group with biotinylated dextran amine (BDA; Sigma-Aldrich, St. Louis, MO). We iontophoresed BDA into the RVLM of the second group. 


\section{CORTICOSPINAL TRACT SPROUTING AFTER SPINAL LESIONS}

After making a midline incision of the scalp, we drilled a $2.0-\mathrm{mm}^{2}$ craniotomy centered $1.0 \mathrm{~mm}$ to the right of the midline and $0.5 \mathrm{~mm}$ caudal to the bregma. After reflecting the dura, we made six to eight pressure injections (total volume $6-8 \mu \mathrm{L}$ ) of $10 \%$ BDA in distilled water at depths of $1 \mathrm{~mm}$ from the cortical surface into the sensorimotor cortex (Li et al., 1990; Paxinos and Watson, 1996) using glass micropipettes (tip diameter, 30-40 $\mu \mathrm{m})$. The scalp was sutured. Before recovery from anesthesia, we treated all rats with an antibiotic and analgesic as described above.

To iontophorese BDA into the RVLM, we flexed the heads of rats ventrally to $-10 \mathrm{~mm}$ relative to the stereotaxic ear bars. The obex was exposed by a dorsal cutaneous incision, reflection of the trapezius muscles and an occipital craniotomy. Using the obex as our stereotaxic reference, we directed a glass micropipette filled with $10 \%$ BDA to a site $1.9 \mathrm{~mm}$ left of the midline, $1.8 \mathrm{~mm}$ rostral to the obex, and $1.8 \mathrm{~mm}$ ventral to the obex. We delivered BDA by iontophoresis (3 microamperes for 20-25 min, 7-sec on/off cycle). Pipettes were left in place for $5 \mathrm{~min}$ after cessation of iontophoresis to reduce leakage along the pipette tract.

\section{Identification of SPN}

We identified SPN by their uptake of Fluorogold ${ }^{\circledR}$ (FG; Fluorochrome, Inc, Denver, CO) administered intraperitoneally (ip). One week after we injected BDA into lesioned rats and 2 weeks before sacrifice of control rats, we anesthetized rats briefly with halothane and injected $3 \mathrm{mg}$ of FG in distilled water i.p. This procedure reliably labels all SPN (Anderson and Edwards, 1994).

\section{Infection with Pseudorabies Virus}

Renally injected PRV infected renal postganglionic neurons and then the SPN that synapsed on those renal postganglionic neurons. IN that synapsed on infected SPN became, in turn, infected. Therefore, we identified neurons that were labeled for both FG and PRV as renally infected SPN. Neurons that were labeled for PRV alone were identified as sympathetically related IN. Five days after injection with FG, we reanesthetized both lesioned and control rats with halothane. A left flank laparotomy was made to expose the left kidney. We made one injection each $(0.5 \mu \mathrm{L})$ of the Bartha strain of pseudorabies virus (PRV, $1 \times 10^{8} \mathrm{PFU} / \mathrm{mL}$, kindly supplied by Dr. Arthur D. Loewy, Washington University, St. Louis, MO) at the cranial and caudal poles of the kidney. Care was taken to prevent exposure of surrounding tissue to PRV. Renal injection sites were sealed with a drop of cyanoacrylate, and the muscle and skin incisions were closed separately. Antibiotics and analgesics were administered as described above. Rats were observed carefully several times each day to ensure that they showed no signs of discomfort. Rats ate, drank, and gained weight normally during this post-infection survival period.

Data from this and other laboratories indicate that the degree of PRV infection is closely related to the volume and site of injection and to survival time (Schramm et al., 1993; Tang et al., 2003; Cano et al., 2004). Our criteria for an "appropriate" degree of infection were that both SPN and IN were infected, but rats exhibited neither infected alpha-motoneurons nor infected SPN on the side contralateral to the renal injection. Based on these criteria, we chose a 73-h survival period for all of the rats in this study.

\section{Tissue Preparation}

At $73 \mathrm{~h}$ after infection, rats were reanesthetized with halothane and injected with $1 \mathrm{~g}$ of urethane dissolved in water to sustain anesthesia during perfusion. We perfused rats transcardially with $200 \mathrm{~mL}$ of physiological saline followed by $500 \mathrm{~mL}$ of $4 \%$ paraformaldehyde in $0.1 \mathrm{M}$ phosphate buffer (PB). Spinal cords and brains were removed and post-fixed in the perfusion fixative overnight, and transferred to $30 \%$ sucrose in $0.1 \mathrm{M}$ PB until they no longer floated.

Our previous work indicated that PRV-infected neurons were concentrated in the $T_{8}$ to $T_{12}$ segments, with peaks at $\mathrm{T}_{10-12}$ (Schramm et al., 1993; Tang et al., 2003). Therefore, we cut $40-\mu \mathrm{m}$ sections of a $\mathrm{T}_{8-12}$ block of spinal cord with a freezing microtome, beginning at the surface of the dorsal columns and ending at the disappearance of the ventral horn. Sections were collected serially in 0.1 M PBS and processed for staining in a 96well culture plate. Twenty-micron transverse sections of the $\mathrm{T}_{6-7}$ segments were also cut and used to determine the total numbers of BDA-labeled CST or RVLM axons in each rat. We cut serial, horizontal, 40- $\mu \mathrm{m}$ sections of the $T_{13}$ to $L_{4}$ spinal cord to evaluate the extent of lesions.

\section{Immunohistochemistry}

Sections were rinsed three times in 0.1 M PBS and incubated with agitation for $72 \mathrm{~h}$ at $4^{\circ} \mathrm{C}$ in a solution containing an antibody to PRV raised in pig (1:10,000; kindly provided by Dr. Arthur Loewy, Washington University, St. Louis, MO), 5\% normal goat serum, and 0.3\% Triton-X. Sections were rinsed three times in PBS and incubated for $2 \mathrm{~h}$ in a mixture of goat anti-pig-Cy3 to visualize PRV (1:200; Jackson Immuno Laboratories, West 
PAN ET AL.

Grove, $\mathrm{PA}$ ) and straptavidin-conjugated $\mathrm{Cy} 2$ to visualize the BDA-labeled axons (1:200; Jackson Immuno Laboratories). Sections were washed three times in PBS, mounted on slides, and coverslipped.

We cut coronal sections of the sensorimotor cortex and the RVLM to locate BDA injection sites. We double-labeled sections of the RVLM for tyrosine hydroxylase (TH) and BDA to precisely identify the injection site. Brainstem sections containing the RVLM were incubated overnight in rabbit polyclonal antibody against TH (Chemicon, Temecula, CA) at 1:3000. After three rinses in PBS, sections were incubated in a solution containing straptavidin-conjugated Cy2 (1:200; Jackson Immuno Laboratories) and an anti-rabbit antibodyconjugated Cy3 (1:200).

\section{Extent of Lesions}

The extent of lesions was determined in two ways. First, under the microscope at $\times 10$ and $\times 20$, the broadest extent of each lesion was reconstructed from the original horizontal sections onto a drawing of a representative transverse section of lumbar spinal cord. Damage to both gray and white matter was considered. Second, BDA-labeled axons in either the corticospinal tracts or among axons descending from the RVLM were studied above and below lesions. A pathway was not considered completely destroyed if we observed any of its labeled axons caudal to the lesions.

\section{Quantification of Sprouting}

We quantified BDA-labeled axon collaterals by counting all labeled fibers crossing two lines drawn mediolaterally across the spinal gray matter. These lines were located 3 and $8 \mathrm{~mm}$, respectively, rostral to the caudal end of each horizontal section from which we obtained data on close appositions (caudal $\mathrm{T}_{12}$ or rostral $\mathrm{T}_{13}$, henceforth referred to as $\mathrm{T}_{13}$ ).

\section{Number and Identity of Labeled Neurons}

We identified neurons labeled for FG and infected with PRV as SPN. We identified neurons labeled for PRV infection only (FG-negative) as IN. We identified FG-labeled neurons not infected with PRV as SPN that did not project to renal postganglionic neurons or that projected to renal postganglionic neurons that had escaped infection. Because our only criterion for identifying IN was that they were infected with PRV but not labeled with FG, to ensure a coherent study population, we also considered only SPN infected with PRV. We examined all sections using either an Olympus BH2 or a Nikon Eclipse $80 \mathrm{i}$ microscope.

\section{Close Appositions}

Although we did not attempt to identify synapses using electron microscopy, we inferred putative synapses as close appositions between axons of descending BDAlabeled axons and the somas and proximal dendrites of infected SPN and IN. Appositions were studied with either a $\times 40$ objective or a $\times 100$ oil-immersion objective, and they were manifested by well-defined varicosities apparently lying directly on the apposed neuron. To be considered apposed by an axon, a neuron needed to exhibit only one, well-characterized, axonal varicosity on its soma or one of its dendrites.

We inferred the existence of synapses by close appositions rather then by electron microscopic examination for two reasons. First, although significant numbers of SPN and IN received synapses from CST and RVLM axons, these synapses were sparse enough that we would have had to examine serial sections of all neurons to determine whether they, in fact, received such input. Second, we would have had to detect at the electron microscopic level anterograde labeling of synapses of the CST, immunohistochemical labeling of PRV, and (to differentiate between SPN and IN) immunohistochemical labeling of a marker for SPN (either choline acetyltransferase or FG).

\section{Normalization}

We normalized the incidence of closely apposed SPN and IN in two ways. First, to account for inter-rat variations in the numbers of infected SPN and IN, we expressed data in terms of the percentage of apposed, PRVinfected SPN and IN to the total number of infected SPN and IN, respectively. Second, to account for inter-rat variations in the number of anterogradely labeled axons, we modified slightly the normalization method of Kerschensteiner et al. (2004). We assumed that the number of collaterals and the number of close appositions observed in each rat was proportional to the number of axons that descended from either the cortex or the RVLM. Therefore, in addition to expressing our data as the number of axon collaterals counted in each rat and the percentage of PRV-infected SPN and IN closely apposed by descending axons, we further normalized these data by the average number of descending axons obtained from three to five $T_{6}$, transverse sections chosen at random. We used the number of axons at the $T_{6}$ level because it was sufficiently rostral that it would not have been affected by CST axonal die-back caused by the lesion. We called these normalized data the "sprouting index" and the "apposition index." The sprouting index was computed as the number of collaterals counted 3 and $8 \mathrm{~mm}$ rostral to $T_{13}$, divided by the average number of axons counted in transverse sections at $\mathrm{T}_{6}$. The apposition in- 


\section{CORTICOSPINAL TRACT SPROUTING AFTER SPINAL LESIONS}
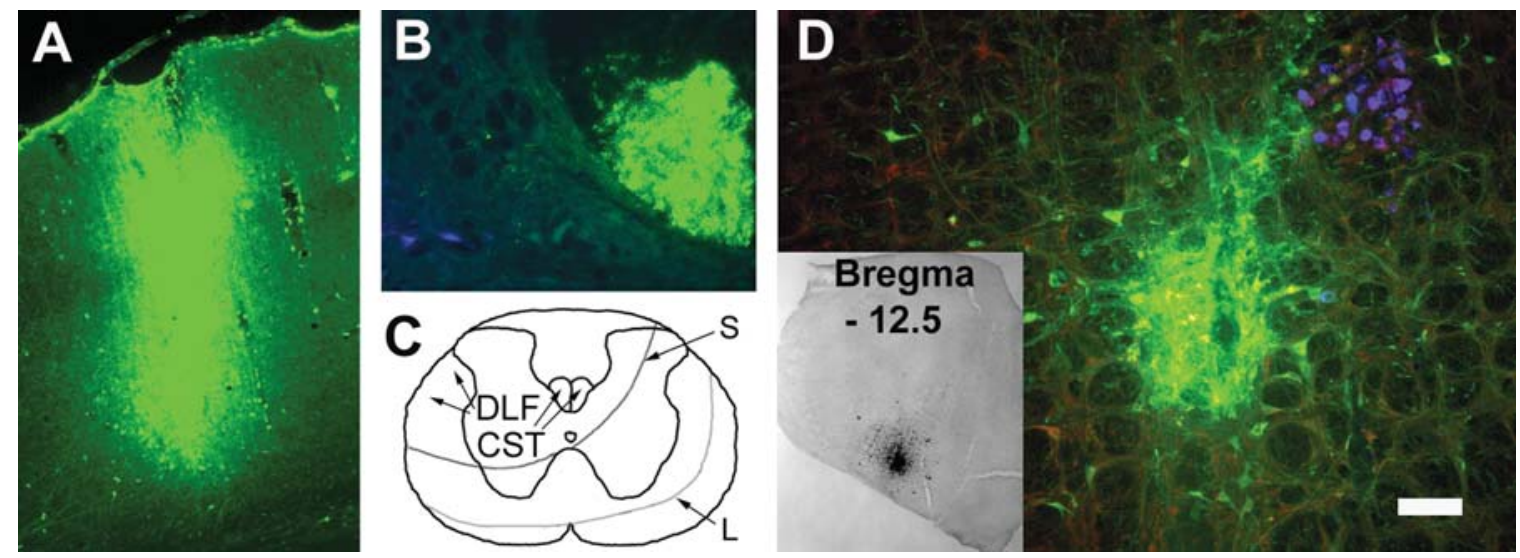

FIG. 1. (A) Coronal section of sensorimotor cortex showing one of eight BDA injection sites (green). (B) Portion of transverse section of thoracic spinal cord. Green, dorsal CST and collaterals; blue, SPN in intercalated area. Note paucity of CST collaterals among SPN. (C) Lumbar spinal section illustrating the extents of the smallest (S) and largest (L) spinal lesions. Left and right corticospinal tracts (CST) and the left dorsolateral funiculus (DLF) are indicated by arrows. Lesions destroyed both left and right CST and the left DLF in all experimental rats. The dorsolateral funiculi contain many descending autonomic pathways. (D) Site of iontophoresis of BDA (green) in the rostral ventrolateral medulla. Blue, nucleus ambiguous (labeled with ip FG). Red, THcontaining neurons of the $\mathrm{C} 1$ cell group (labeled immunohistochemically). Yellow, TH-containing neurons of the $\mathrm{C} 1$ cell group co-labeled with BDA. Bar $=100 \mu \mathrm{m}$. (Inset) Iontophoretic site at low power.

dex was computed as the percentage of PRV-infected SPN and IN closely apposed by descending axons divided by the average number of axons counted in transverse sections at $\mathrm{T}_{6}$.

\section{Statistical Analysis}

Group differences were compared by one-way ANOVA, followed by the Bonferroni multiple comparison test. Data are presented as mean \pm 1 SD.

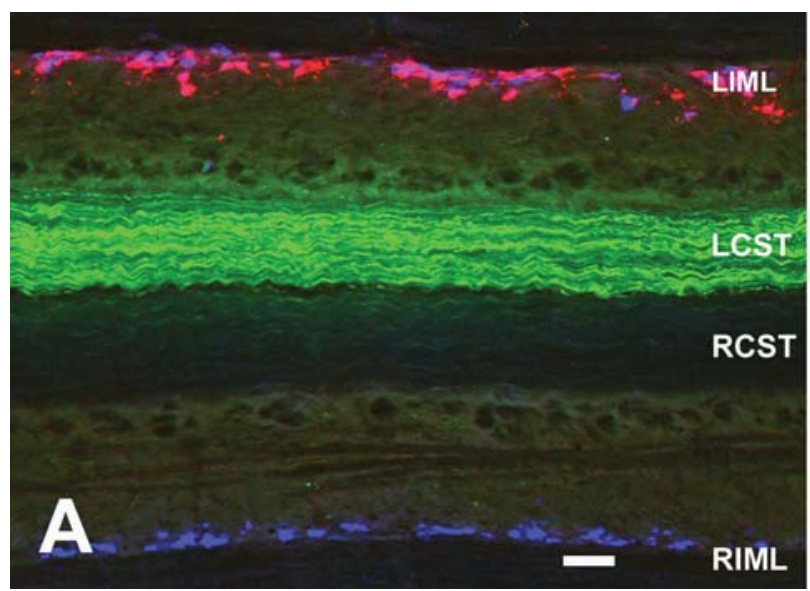

\section{RESULTS}

\section{BDA Injection Sites in the Sensorimotor Cortex and the RVLM}

Cortical injection sites covered a region of roughly $1.0 \times 1.0 \mathrm{~mm}^{2}$, which included the region of primary sensorimotor cortex devoted to the hindlimb, trunk, and forelimb. Most injections included layers IV-V, including the pyramidal neurons from which the axons of the CST are derived (Fig. 1A). In the RVLM, BDA deposition was cen-

FIG. 2. (A) Horizontal section through intermediate zone of $T_{10}$ spinal cord. LIML, left intermediolateral column; LCST, left corticospinal tract, labeled with BDA from right sensorimotor cortex; RCST, right corticospinal tract, unlabeled; RIML, right intermediolateral column. Bar $=200 \mu \mathrm{m}$. (B) Higher-magnification view of left intermediolateral column. CST, corticospinal tract collaterals (green); IN, PRV-infected interneuron (red); ISPN, infected SPN (magenta); USPN, uninfected SPN (blue). Bar $=50 \mu \mathrm{m}$. 
tered near the $\mathrm{C} 1$ cell group (Fig. 1D), caudal to the facial nucleus and ventral to the nucleus ambiguus (NA). Some TH-labeled neurons were double-labeled for BDA in the injection site. The BDA deposit site consisted of a homogeneous compact core surrounded by a halo in which dense and lighter zones were intermingled. Additional confirmation of the location of BDA injections was provided by the labeling of the NA by our i.p. administration of FG (Fig. 1D).

\section{Spinal Lesions}

In all rats, dorsal hemisections destroyed the dorsal columns, both right and left dorsal CST, the left dorsolateral funiculus, the left dorsal horn, and the left intermediate zone (Fig. 1C). The left and right ventrolateral funiculi, ventral corticospinal tracts, right dorsal and ventral horns, and right dorsolateral funiculus were spared to varying degrees among rats. Axons descending from the left RVLM were completely destroyed in all rats. Some crossed axons from the RVLM were observed on the right side of the spinal cord.

\section{Retrogradely Labeled Sympathetic Preganglionic Neurons}

The $\mathrm{T}_{8-12}$ spinal segments of all rats whose left kidneys were injected with PRV were examined to determine their degree of infection. Following a 73-h survival period, 14 rats were judged to be over-infected and 25 rats under-infected. Those rats were not considered further. Of the rats with appropriate PRV infections, the BDA labeling from five cortical injections and five RVLM injections of the control group, and five cortical injections and three RVLM injections of the SCI group, were considered adequate for quantification of close appositions.Consistent with previous observations (Schramm et al., 1993; Tang et al., 2004), PRV-immunoreactive neurons were found throughout $\mathrm{T}_{8-12}$ spinal horizontal sections with a maximum density between $\mathrm{T}_{10}$ and $\mathrm{T}_{11}$. Infected neurons were concentrated within the intermediolateral column (IML) ipsilateral to the infected kidney (Fig. 2A). However, infected neurons were also found in the lateral funiculus (LF), central autonomic area (CA), the intercalated area (IC), and in dorsal horn laminae IV-VI of the injected side (for description of regions, see Schramm, et al., 1993). Smaller numbers of infected neurons were located in dorsal horn laminae I-III.

The majority of the infected SPN were located in clusters in the IML and intermingled with non-infected SPN (Fig. 2A, B). Infected IN were located in the IML and the IC, and most of them were in close proximity to SPN. As noted by Tang et al. (2004), the locations of clusters of IN were closely correlated with clusters of SPN. We also observed infected IN in dorsal horn laminae III-VI. Very few infected IN were observed in the LF and in laminae I-II. IN were frequently smaller than SPN.

\section{CST Projections}

Cortical injection of BDA yielded intense labeling of the left CST (Figs. 1B and 2A). A small number of BDAlabeled CST fibers also were found in the right dorsolateral and ventral funiculi. In $\mathrm{T}_{8}-\mathrm{T}_{12}$ horizontal sections, we observed many BDA-labeled collaterals leaving the CST and coursing laterally into the medial portions of the deep dorsal horn laminae and intermediate zone. Most of the collaterals were derived from CST axons in the dorsal funiculus. This division of the CST contains more than $95 \%$ of CST fibers (Weidner et al., 2001).
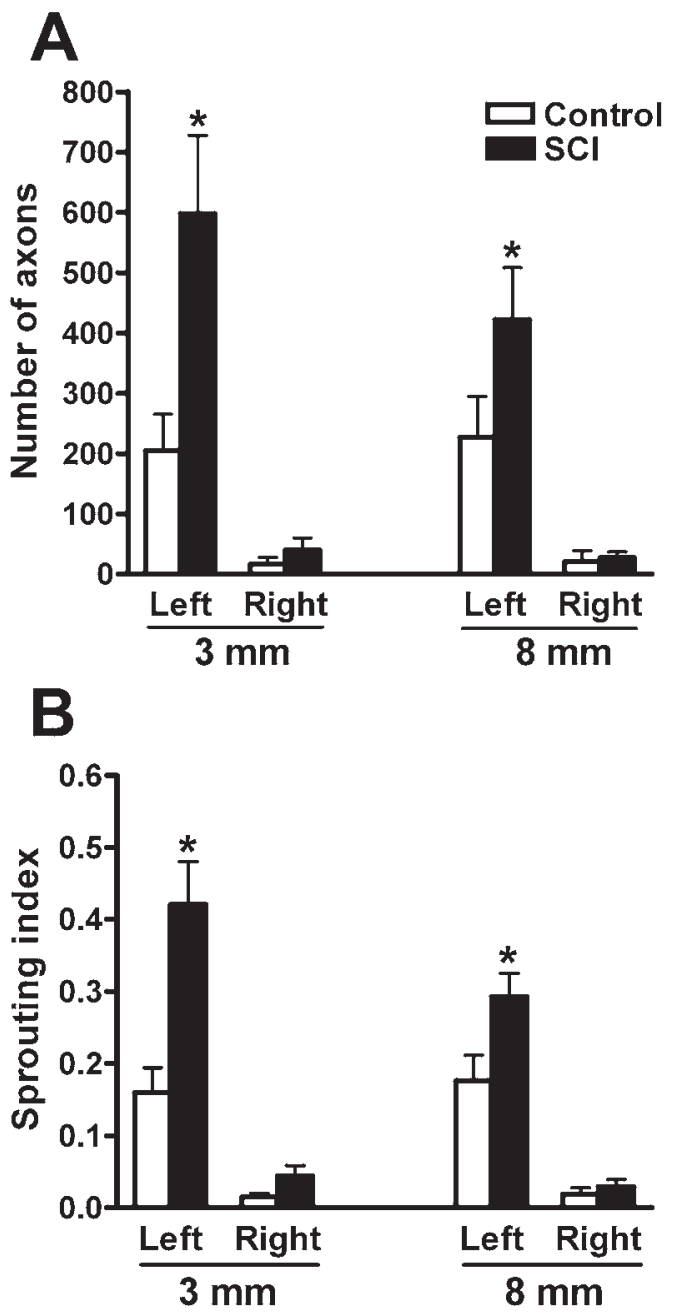

FIG. 3. (A) Numbers of labeled CST collaterals 3 and $8 \mathrm{~mm}$ rostral to $\mathrm{T}_{13}$. (B) CST sprouting index (number of labeled collaterals normalized by the number of labeled, descending, CST axons in each rat). ${ }^{*} p<0.001$. 


\section{CORTICOSPINAL TRACT SPROUTING AFTER SPINAL LESIONS}

In all rats, the region of heaviest collateralization was the medial half of laminae IV-VI. Some labeled axons were also present in lamina $\mathrm{X}$, the medial half of lamina VII, and the dorsomedial half of lamina VIII. However, few BDAlabeled axons reached the IML, and those axons that did tended to ramify between, rather than within, clusters of SPN (Figs. 2B and 4A). We observed few labeled CST axons in the gray matter of the right thoracic spinal cord.

After SCI, we observed significantly more BDA-labeled CST axon collaterals on the lesioned side of the spinal cord both 3 and $8 \mathrm{~mm}$ rostral to $\mathrm{T}_{13}$ than in rats with intact spinal cords (Fig. 3). Three millimeters rostral to $\mathrm{T}_{13}$ in SCI rats, we observed $598 \pm 130$ and $40 \pm$ 20 collaterals on the left and right sides of the spinal cord, respectively. Eight millimeters rostral to $\mathrm{T}_{13}$, we observed $422 \pm 87$ and $28 \pm 9$ collaterals on the left and right sides, respectively. Three millimeters rostral to $T_{13}$ in control rats, we observed $204 \pm 61$ and $17 \pm 11$ collaterals on the left and right sides of the spinal cord, respectively. Eight millimeters rostral to $\mathrm{T}_{13}$, we observed $227 \pm 68$ and $21 \pm 18$ collaterals on the left and right sides, respectively ( $p<0.001$ for the differences in numbers of collaterals between SCI and control rats).

The diameters of many of the CST sprouts in lesioned rats appeared to be smaller than those of axons in control rats (Fig. 4). These collaterals were distributed throughout laminae II-VII, and many reached the IML and the lateral dorsal horn. Many CST collaterals with varicosities were intermingled within clusters of SPN, and many SPN were closely apposed by BDA labeled axon profiles (Fig. 4B).
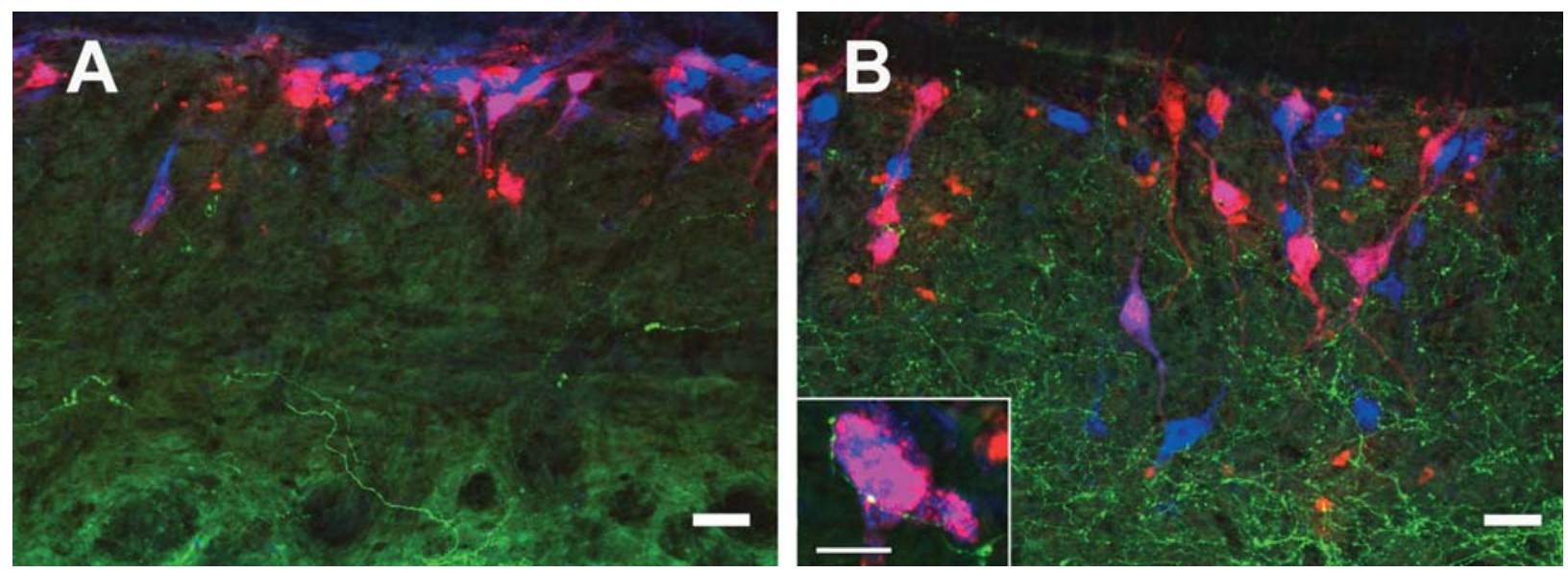

FIG. 4. (A) Horizontal section of left IML of rat with intact spinal cord (labeling as in Fig. 2B). Bar $=50 \mu \mathrm{m}$. Note paucity of CST collaterals and failure of CST collaterals to closely appose either SPN or IN. (B) Horizontal section of left IML of SCI rat. Note both the high concentration of CST collaterals among sympathetic neurons and the incidence of multiple CST axonal varicosities on sympathetic neurons. Bar $=200 \mu \mathrm{m}$. (Inset) High magnification photograph of close apposition between a CST axon and an infected SPN in an SCI rat. Bar $=25 \mu \mathrm{m}$. 

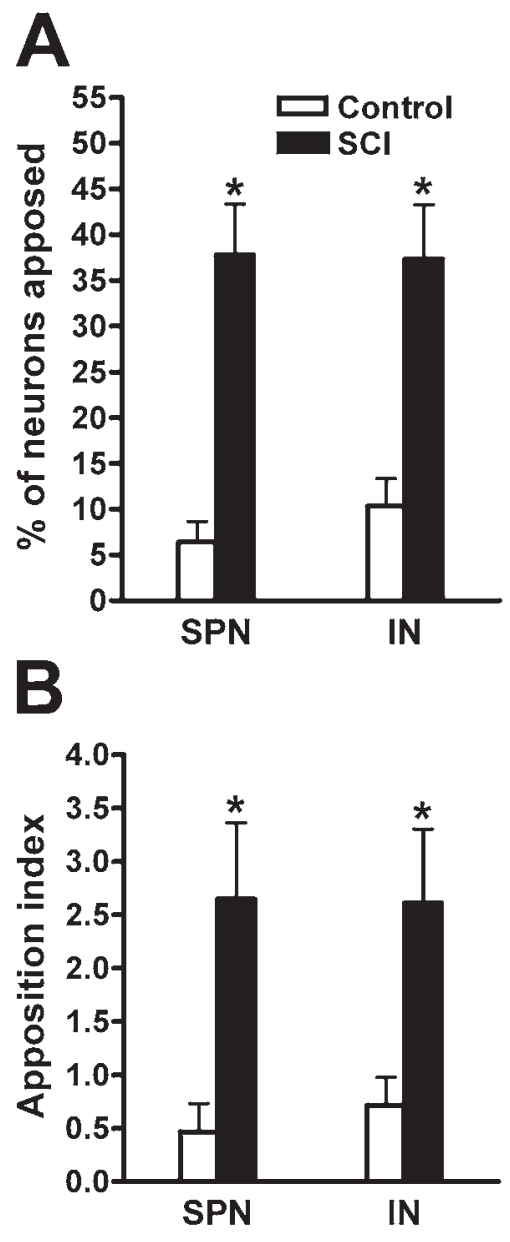

FIG. 5. (A) Percentage of infected SPN and IN closely apposed by CST collaterals in uninjured and SCI rats. (B) Apposition index (percentage of infected SPN and IN closely apposed by CST collaterals normalized by the number of labeled, descending, CST axons in each rat) in uninjured and SCI rats. $* p<0.001$.

left side (ipsilateral to the injection). Collaterals frequently emerged from labeled axons in the DLF and entered the IML. The majority of these collaterals sprouted within the IML and appeared to be concentrated within clusters of SPN. Some collaterals and branches extended to lamina $\mathrm{X}$, and a few projected to the right (contralateral) IML. We also observed axons in the LF, IC, and $\mathrm{CA}$ regions. A few collaterals projected to the ventral horn and to the lateral part of laminae V-VI.

The numbers of collaterals of RVLM axons observed in SCI rats were similar to those observed in control rats, both 3 and $8 \mathrm{~mm}$ rostral to the $\mathrm{T}_{13}$ segment. At 3 $\mathrm{mm}$, the average numbers of collaterals for control rats were $47.8 \pm 20.3$ and $17.7 \pm 6.1$, for left and right sides, respectively. The numbers of collaterals for SCI rats were $49.3 \pm 25.5$ and $11.3 \pm 4.5$, for the left and right sides, respectively. At $8 \mathrm{~mm}$, the numbers of collaterals for control rats were $63.5 \pm 37.3$ and $20.0 \pm$ 12.3 , for left and right sides, respectively. Numbers of collaterals were $61.3 \pm 26.2$ and $15.0 \pm 8.8$, for left and right sides, respectively in SCI rats (differences between SCI and control rats, $p>0.05$ for both left and right sides).

Collaterals of RVLM axons in the DLF gave rise to numerous branches that closely intertwined clusters of infected SPN and closely apposed SPN at multiple sites (Fig. 6). RVLM axons also closely apposed non-infected SPN, but these appositions were not quantified. Many more SPN than IN were closely apposed by RVLM axons in both control and SCI rats. In control rats, $20.8 \pm$ $2.5 \%$ percent of infected SPN were apposed by RVLM axons. However, fewer infected IN $(5 \pm 0.9 \%)$ were apposed by RVLM axons. In SCI rats, $30.2 \pm 7.5 \%$ of infected SPN were apposed by RVLM axons. Similarly, fewer infected IN $(6.8 \pm 1.6 \%)$ were apposed by RVLM axons ( $p<0.0001$ for difference between RVLM axonal appositions on SPN and IN). Although, on average, RVLM axons exhibited more close appositions with SPN and IN in SCI rats than in control rats, this difference was not statistically significant.

\section{DISCUSSION}

Although this study investigated collateralizing axons rather than regenerating axons per se, regenerating axons would, by definition, be collaterals of existing descending tracts. The rationale of this study was that similar synapses would be made by collaterals of axons of sprouting, descending pathways, whether these collaterals were rostral or caudal to a lesion. Therefore, our observation that axons of the CST closely approximate many more SPN and IN in SCI rats than in rats with intact spinal cords suggests that, via post-injury plasticity, spinal sympathetic neurons could receive inappropriate descending inputs.

The number of rats available for analysis in this study was relatively small for two reasons. First, as expected, we experienced some mortality among rats with spinal cord lesions. Second, we required the success of several relatively capricious methods to obtain each useable rat. In our hands, the intensity of anterograde labeling with BDA varies considerably between rats. The brightness of labeling of SPN using FG injections also varies. Using a 73-h survival time, SPN but not IN were observed in lightly infected rats, and substantial numbers of alphamotoneurons were labeled in heavily infected rats. Thus, in combination with the requirement for good BDA and 


\section{CORTICOSPINAL TRACT SPROUTING AFTER SPINAL LESIONS}
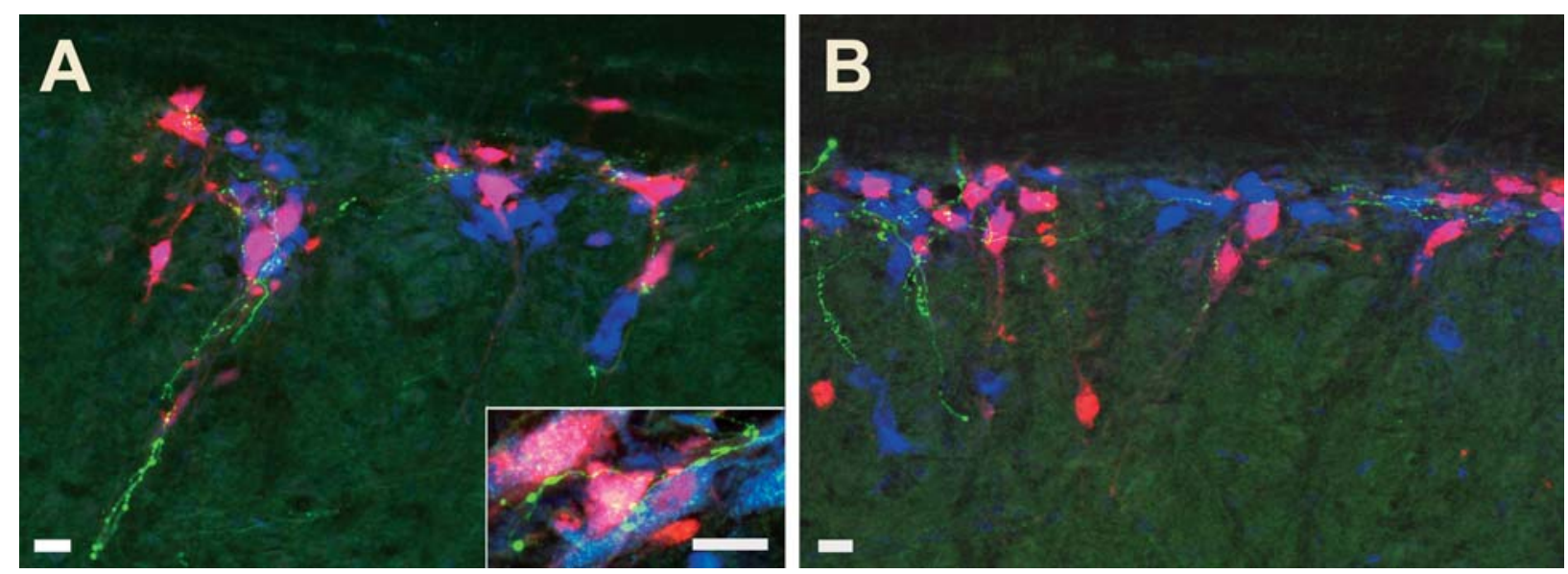

FIG. 6. (A) Appositions between labeled RVLM axons in uninjured rat. (Inset) Higher magnification. (B) Appositions between labeled RVLM axons in SCI rat. Labeling as in Figure 2B, except that green axons are from RVLM instead of cortex. Note similarity between numbers and morphologies of collaterals and appositions in SCI and uninjured rats. Bar $=25 \mu \mathrm{m}$.

FG labeling, our strict criteria for the degree of PRV infection reduced our yield of useable rats to 18 .

As discussed above, we were forced by technical considerations to assume that close appositions between the axons of descending pathways and the somas and dendrites of retrogradely labeled spinal sympathetic neurons represented putative synapses. Although open to interpretation, this assumption is supported by previous studies (Zagon and Smith, 1993; Llewellyn-Smith et al., 2003).

\section{CST Projections to SPN and Spinal IN}

Our results represent the first observations of close appositions between the axons of cortical neurons and SPN and between cortical neurons and sympathetically related spinal IN. Several previous reports have described sensorimotor cortical projections to the intermediate zone of the spinal cord (Petras, 1967; Armand and Kuypers, 1980; Martin, 1996; Yang and Lemon, 2003). However, the putative targets of those projections were not determined.

To achieve the broadest population of projections, we attempted to include in our injection sites as much of the sensorimotor cortex as possible (Miller, 1987). In rats with intact spinal cords, collaterals of CST axons projected into medial regions of the dorsal horn, where Chau et al. (1997, 2000) identified a small number of neurons whose activities were positively correlated with renal sympathetic nerve activity (RSNA). However, collaterals of few CST axons projected laterally enough that they entered the intermediolateral column, where the greatest concentration of sympathetically correlated neurons resided and where Tang et al. (2004) localized the largest concentration of IN labeled after renal PRV injections.

Confirming previous reports (Fouad et al., 2001; Weidner et al., 2001; Raineteau et al., 2002), spinal injury elicited substantial sprouting of the corticospinal tract rostral to the lesion. The trajectories of these collaterals were not limited to regions well supplied with collaterals of the CST in intact rats. Instead, many collaterals projected more laterally, and they closely apposed substantial numbers of SPN and IN. Significantly, not only did the number of approximated SPN and IN increase, but the morphology of those approximations resembled more closely the approximations characteristic of axons from the RVLM, rather than appearing almost accidental, as they did in spinally intact rats.

Many laboratories have described autonomic effects of cortical stimulation or cortical lesions (Hoff et al., 1963; Cechetto and Saper, 1990). That cortical synapses on spinal sympathetic neurons may exist, as indicated by the present study, suggests that the cortex could play a role in directly affecting sympathetic activity in rats with intact spinal cords. However, reviewers have not considered the evidence for such direct effects convincing (Cechetto and Saper, 1990). A more likely mode of autonomic regulation by cerebral cortex (and very likely the mechanism for most previously-reported corticospinal autonomic effects) would seem to be the substantial cortical projections to the hypothalamus and RVLM (Oppenheimer et al., 1992; Sequeira et al., 2000).

We are unable to predict the physiological significance of an increased projection of CST axons to spinal sympathetic neurons following a chronic spinal lesion. If, in fact, our observed approximations represent synapses, 
then more SPN could be excited by cortical inputs after, than before, spinal injury. The physiological effect of increased appositions on IN is unclear because these neurons could either excite or inhibit sympathetic activity. It is worth pointing out, however, that Chau et al. (1997, 2000) and Tang et al. (2003) observed very few IN with activity that was negatively correlated with RSNA in rats with acutely transected spinal cords. Based on that evidence, we suggest that cortical effects on sympathetic activity via IN, as well as directly upon SPN, would favor excitation of sympathetic activity.

\section{RVLM Projections to SPN and Spinal IN}

We traced projections from the RVLM to spinal sympathetic neurons for two reasons. First, although the RVLM has been shown to project monosynaptically to SPN (Milner et al., 1988; Bernstein-Goral and Bohn, 1989; Zagon and Smith, 1993; Pyner and Coote, 1998; Moon et al., 2002), projections to sympathetically related spinal interneurons had not been studied previously. Second, we wanted to determine whether spinal injury altered the incidence of close appositions between RVLM axons and spinal sympathetic neurons.

Confirming previous observations, the BDA-labeled axons of RVLM neurons entered the spinal gray matter from the ipsilateral dorsolateral and lateral funiculi and closely approximated the dendrites and somas of many SPN. Some axons crossed the midline and closely approximated contralateral SPN. This pathway is considered a major source of descending excitation for SPN (Ross et al., 1981; Morrison et al., 1991). Our observation that only $20 \%$ of infected SPN were closely approximated by RVLM axons may have resulted from our restricted iontophoresis of BDA in the RVLM. Alternatively, this relatively sparse projection may be a characteristic of RVLM projections to neurons affecting the kidney.

The morphology of the close approximations between RVLM axons and spinal sympathetic neurons in rats with both lesioned and intact spinal cords differed significantly from those between CST axons and spinal sympathetic neurons in intact rats. In rats with intact spinal cords, approximations with the CST often consisted of one or two varicosities, en passant, as the axon traversed a soma or dendrite. In contrast, the axons of RVLM neurons usually approximated somas and dendrites tangentially, exhibiting many closely apposed varicosities.

Importantly, a substantially larger percentage of SPN than IN was closely apposed by axons projecting from the RVLM. This observation suggests that regulation of sympathetic activity by the RVLM is mediated largely by direct excitation of SPN rather than through excitation of IN. This conclusion is supported by the observation by Miller et al. (2001) that, although the activities of many spinal IN are correlated with RSNA after cervical spinal transection, few sympathetically correlated spinal IN are observed in rats with intact spinal cords. Unlike CST axons, the projections, incidence of close appositions, and morphology of close appositions axons of RVLM axons were not significantly affected by spinal lesions. Failure to observe changes in this population of axons was unlikely to have been caused by incomplete lesions of the dorsolateral funiculus in some rats because no correlation was seen between the sizes and locations of these lesions and the degree of sprouting rostral to the lesions.

In summary, we have shown that in rats with intact spinal cords, CST axons appose a small number of spinal sympathetic neurons. These appositions are often manifested by only one or two varicosities on axons whose trajectories are normal to the dendrites and somas of apposed neurons. However, one month after a spinal lesion, CST axons sprout abundantly and closely appose many more spinal sympathetic neurons. These appositions are manifested by more varicosities on axons whose trajectories are tangential to the dendrites and somas of apposed neurons. Spinal lesions do not similarly affect axons descending from the RVLM and their close appositions with spinal sympathetic neurons. Additional anatomical and physiological experiments will be necessary to determine whether the morphological changes observed in the present experiments are both physiologically significant and sustained over survival periods longer than one month. Nevertheless, these results recommend that autonomic function other than bladder control be assessed in models of spinal cord repair.

\section{ACKNOWLEDGMENTS}

We gratefully acknowledge the technical assistance of Anand Veeravagu. This research was supported by NIH grant HL16315.

\section{REFERENCES}

ANDERSON, C.R., and EDWARDS, S.L. (1994). Intraperitoneal injections of Fluorogold ${ }^{\circledR}$ reliably labels all sympathetic preganglionic neurons in the rat. J. Neurosci. Methods 53, 137-141.

ARMAND, J., and KUYPERS, H.G. (1980). Cells of origin of crossed and uncrossed corticospinal fibers in the cat: a quantitative horseradish peroxidase study. Exp. Brain Res. 40, 23-34.

BAREYRE, F.M., KERSCHENSTEINER, M., RAINETEAU O., METTENLEITER T.C., WEINMANN O., and 


\section{CORTICOSPINAL TRACT SPROUTING AFTER SPINAL LESIONS}

SCHWAB M.E. (2004). The injured spinal cord spontaneously forms a new intraspinal circuit in adult rats. Nat. Neurosci. 7, 269-277.

BERNSTEIN-GORAL, H., and BOHN, M.C. (1989). Phenylethanolamine $\mathrm{N}$-methyltransferase-immunoreactive terminals synapse on adrenal preganglionic neurons in the rat spinal cord. Neuroscience 32, 521-537.

CANO, G., CARD J.P., and SVED, A.F. (2004). Dual viral transneuronal tracing of central autonomic circuits involved in the innervation of the two kidneys in rat. J. Comp. Neurol. 471, 462-481.

CECHETTO, D.F., and SAPER, C.B. (1990). Role of the cerebral cortex in autonomic function, in: Central Regulation of Autonomic Function. A.D. Loewy, and K.M. Spyer (eds), Oxford University Press: New York, pps. 208-223.

CHAU, D., KIM, N., and SCHRAMM, L.P. (1997). Sympathetically correlated activity of dorsal horn neurons in spinally transected rats. J. Neurophysiol. 77, 2966-2974.

CHAU, D., JOHNS, D.G., and SCHRAMM, L.P. (2000). Ongoing and stimulus-evoked activity of sympathetically correlated neurons in the intermediate zone and dorsal horn of acutely spinalized rats. J. Neurophysiol. 83, 2699-2707.

FOUAD, K., PEDERSEN, V., SCHWAB, M.E., and BROSAMLE, C. (2001). Cervical sprouting of corticospinal fibers after thoracic spinal cord injury accompanies shifts in evoked motor responses. Curr. Biol. 11, 1766-1770.

HOFF, E., KELL, J.F., JR., and CARROLL, M.N., JR. (1963). Effects of cortical stimulation and lesions on cardiovascular function. Physiol. Rev. 43, 68-114.

KERSCHENSTEINER, M., BAREYRE, F.M., BUDDEBERG, B.S., et al. (2004). Remodeling of axonal connections contributes to recovery in an animal model of multiple sclerosis. J. Exp. Med. 200, 1027-1038.

LLEWELLYN-SMITH, I.J., MARTIN, C.L., MARCUS, J.N., YANAGISAWA, M., MINSON, J.B., and SCAMMELL, T.E. (2003). Orexin-immunoreactive inputs to rat sympathetic preganglionic neurons. Neurosci. Lett. 351, 115-119.

LLEWELLYN-SMITH, I.J., and WEAVER, L.C. (2001). Changes in synaptic inputs to sympathetic preganglionic neurons after spinal cord injury. J. Comp. Neurol. 435, 226-240.

LI, X.G., FLORENCE, S.L., and KAAS, J.H. (1990). Areal distributions of cortical neurons projecting to different levels of the caudal brain stem and spinal cord in rats. Somatosens Mot. Res. 7, 315-335.

MARTIN, J.H. (1996). Differential spinal projections from the forelimb areas of the rostral and caudal subregions of primary motor cortex in the cat. Exp. Brain Res. 108, 191-205.

MILLER, C.O., JOHNS, D.G., and SCHRAMM, L.P. (2001). Spinal interneurons play a minor role in generating ongoing renal sympathetic nerve activity in spinally intact rats. Brain Res. 918, 101-106.
MILLER M.W. (1987). The origin of corticospinal projection neurons in rat. Exp. Brain Res. 67, 339-351.

MILNER, T.A., MORRISON, S.F., ABATE, C., and REIS, D.J. (1988). Phenylethanolamine $N$-methyltransferase-containing terminals synapse directly on sympathetic preganglionic neurons in the rat. Brain Res. 448, 205-222.

MOON, E.A., GOODCHILD, A.K., and PILOWSKY, P.M. (2002). Lateralisation of projections from the rostral ventrolateral medulla to sympathetic preganglionic neurons in the rat. Brain Res. 929, 181-190.

MORRISON, S.F., CALLAWAY, J., MILNER, T.A., and REIS, D.J. (1991) Rostral ventrolateral medulla: a source of the glutamatergic innervation of the sympathetic intermediolateral nucleus. Brain Res. 562, 126-135.

OPPENHEIMER, S.M., SALEH, T., and CECHETTO, D.F. (1992). Lateral hypothalamic area neurotransmission and neuromodulation of the specific cardiac effects of insular cortex stimulation. Brain Res. 581, 132-142.

PAN, B., KIM, E.J., and SCHRAMM, L.P. (2004). Projections to spinal sympathetic interneurons (IN) and sympathetic preganglionic neurons (SPN) from sensorimotor cortex and rostral ventrolateral medulla (RVLM) in rats. Neurosci. Abst. abstract 74.7 .

PAXINOS, G., and WATSON, C. (1996). The Rat Brain in Stereotaxic Coordinates. Academic Press: New York.

PETRAS, J.M. (1967). Cortical, tectal and tegmental fiber connections in the spinal cord of the cat. Brain Res. 6, 275-324.

PYNER, S., and COOTE, J.H. (1998). Rostroventrolateral medulla neurons preferentially project to target-specified sympathetic preganglionic neurons. Neuroscience 83, 617-631.

RAINETEAU, O., FOUAD, K., BAREYRE, F.M., and SCHWAB, M.E. (2002). Reorganization of descending motor tracts in the rat spinal cord. Eur. J. Neurosci. 16, 1761-1771.

RANSON, R.N., MOTAWEI, K., PYNER, S., and COOTE, J.H. (1998) The paraventricular nucleus of the hypothalamus sends efferents to the spinal cord of the rat that closely appose sympathetic preganglionic neurones projecting to the stellate ganglion. Exp. Brain Res. 120, 164-172.

RAMER, L.M., RAMER, M.S., and STEEVES, J.D. (2005). Setting the stage for functional repair of spinal cord injuries: a cast of thousands. Spinal Cord 43, 134-161.

ROSS, C.A., ARMSTRONG, D.M., RUGGIERO, D.A., PICKEL, V.M., JOH, T.H., and REIS, D.J. (1981). Adrenaline neurons in the rostral ventrolateral medulla innervate thoracic spinal cord: a combined immunocytochemical and retrograde transport demonstration. Neurosci. Lett. 25, 257-262.

SCHRAMM, L.P., STRACK, A.M., PLATT, K.B., and LOEWY, A.D. (1993). Peripheral and central pathways reg- 


\section{PAN ET AL.}

ulating the kidney: a study using pseudorabies virus. Brain Res. 616, 251-262.

SEQUEIRA, H., VILTART, O., BA-M'HAMED, S., and PAULAIN, P. (2000). Cortical control of somato-cardiovascular integration: neuroanatomical studies. Brain Res. Bull. 53, 87-93.

TANG, X., NECKEL, N.D., and SCHRAMM, L.P. (2003) Locations and morphologies of sympathetically correlated neurons in the $\mathrm{T}(10)$ spinal segment of the rat. Brain Res. 976, 185-193.

TANG, X., NECKEL, N.D., and SCHRAMM, L.P. (2004). Spinal interneurons infected by renal injection of pseudorabies virus in the rat. Brain Res. 1004, 1-7.

WEIDNER, N., NER, A., SALIMI, N., and TUSZYNSKI, M.H. (2001). Spontaneous corticospinal axonal plasticity and functional recovery after adult central nervous system injury. Proc. Natl. Acad. Sci. USA 98, 3513-3518.
YANG, H.W., and LEMON, R.N. (2003). An electron microscopic examination of the corticospinal projection to the cervical spinal cord in the rat: lack of evidence for cortico-motoneuronal synapses. Exp. Brain Res. 149, 458-69.

ZAGON, A., and SMITH, A.D. (1993). Monosynaptic projections from the rostral ventrolateral medulla oblongata to identified sympathetic preganglionic neurons. Neuroscience 54, 729-743.

Address reprint requests to: Baohan Pan, Ph.D. The Johns Hopkins School of Medicine 605 Traylor Building 720 Rutland Ave. Baltimore, MD 21205

E-mail: bpan2@jhmi.edu 